\title{
Surgical Treatment of Displaced Abomasum in Cattle Using Ljubljana Method
}

\author{
Jože Staričํ, Halil Selcuk Biricik², Gurbuz Aksoy ${ }^{3}$, Tomaž Zadnik ${ }^{1}$ \\ ${ }^{1}$ Clinic for Ruminants, Veterinary Faculty, University of Ljubljana, Slovenia \\ ${ }^{2}$ Department of Surgery, ${ }^{3}$ Department of Internal Medicine, Faculty of Veterinary Medicine, \\ Harran University, Turkey
}

Received April 5, 2009

Accepted May 13, 2010

\begin{abstract}
Displacement of the abomasum (DA) is an increasingly common disease in dairy cattle in intensive production. This study presents surgical treatment of DA using Ljubljana method in years 2005 and 2006. Slovenian Black and White dairy cows $(n=30)$ with left DA in 19 cows, right DA in four cows, anterior DA in two cows and abomasal volvulus in five cows were used. The surgical treatment was successful in 28 cows, 2 had to be euthanized due to complications associated with left DA and abomasal volvulus. One month after the surgery all 28 cows were productive. Omentopexy using Ljubljana method can be used as a clinical procedure as well as procedure performed in ambulatory practice.
\end{abstract}

Displacement, surgery, percutaneous, abdomen, omentopexy

In the last decade, displacement of the abomasum (DA) has been one of the most common surgical interventions in dairy cattle. It occurs due to gas accumulation in the abomasum, which pulls the organ from its physiological position on the abdominal floor either between the left abdominal wall and rumen or between the right abdominal wall and intestines. Distended abomasum can also change its normal position in the cranial direction. In such case, displaced abomasum is trapped between the reticulum and diaphragm. This type of displacement is called anterior DA (Zadnik et al. 2001; Zadnik 2003a). The incidence of left abomasal displacement on lactation varies between 0.5 and $2.2 \%$; in certain circumstances it may be even 5\% or more (Steiner 2006). Left DA is much more common (85-96\% of DA) than right DA, accounting for 4-15\% of cases (Constable et al. 1992). In a study conducted in Slovenia, anterior abomasal displacement accounted for $12.4 \%$ of cases (Zadnik 2003a).

Majority of cases develop within the first month after calving and other cases develop during last months of pregnancy or in other physiological periods. Beside known predisposing factors such as negative energy balance, hypocalcaemia, retained foetal membranes, uterine infections, dystocia, cow breed, and others, physiologically more transverse and cranial position of the abomasum during the last 3 months of gestation (Wittek et al. 2005) and increasing depth of abdomen (Wittek et al. 2007) increase the risk of displacement in dairy cattle.

In rare cases, abomasum spontaneously returns to its physiological position on the abdominal floor. In majority of cases, treatment of the affected animal is necessary. Decision upon the type of treatment is dependent on various factors including availability of equipment, clinical status of the animal, costs of procedure, clinician's skills and preferences, value of the animal, owner's wishes, etc. Abomasal displacements can be corrected by surgical or nonsurgical techniques. Nonsurgical techniques include rolling the cow to flip the abomasum back in place, or securing the abomasum to the body wall with a percutaneous blind tack or a toggle pin fixation. Rolling is not an effective long-term treatment because most cows will have a recurrence. Left DA can be corrected surgically using right flank omentopexy, right paramedian abomasopexy, left flank abomasopexy, 
one-step standing or dorsally recumbent cow laparoscopic abomasopexy or two-step laparoscopic abomasopexy. Right DA and abomasal volvulus are corrected surgically using right paralumbar fossa omentopexy or right paramedian abomasopexy. Current scientific findings favour laparoscopy-assisted correction of uncomplicated left DA over other surgical procedures due to lesser invasiveness, quicker completion of surgery, less complications, quicker return to productivity and minor or no need of antibacterial treatment (Rohn et al. 2004; Seeger et al. 2006; Steiner 2006; Roy et al. 2008).

At the Clinic for Ruminants, Veterinary Faculty of Ljubljana we generally advise surgical correction in the right paralumbar fossa based on more than 30-year-long experience with DA. Right paralumbar fossa laparotomy gives excellent opportunity for evaluation of almost the whole abdomen with organs, very good orientation and possibility of favourable omentopexy position. Based on obtained information about clinical status, surgical and clinicopathological findings, we are able to institute post-surgical medical treatment, focused also on other pathological findings obtained at surgery and to give a firm prognosis about recovery of the affected cow (Zadnik et al. 2001; Zadnik 2003b).

The study describes Ljubljana method of surgical correction of all types of displacements of the abomasum or abomasal volvulus.

\section{Materials and Methods}

Animals

A total of 30 Slovenian Black and White dairy cows were included in this study. All the cows were within 4 weeks after calving, except one cow that was 140 days in milk. Cows were affected with left displacement of abomasum (19), right DA (4), anterior DA (2) and abomasal volvulus (5).Cows were surgically treated by Ljubljana method at the Clinic for Ruminants, Veterinary Faculty of Ljubljana in the years 2005 and 2006.

Diagnosis

The diagnosis was based on findings obtained by signalment and history of the disease and clinical examination. The diagnosis was confirmed surgically. Clinic-pathological profile was examined to better assess the health status of affected cows and further assist them with specific treatments (supplementation of fluids, minerals, glucose, etc.) to facilitate faster return to full production.

Anaesthesia

Local anaesthesia with $2 \%$ procaine (procaine powder with sterile distilled water) was prepared. Proximal paralumbar block (last thoracic T 13 and first two lumbar L 1 and L 2 spinal nerves) and inverted L block a few $\mathrm{cm}$ cranial from surgical incision was performed. Twelve $\mathrm{ml}$ of $2 \%$ procaine were injected at each location of dorsal and ventral branch of spinal nerves. Additional $60 \mathrm{ml}$ was infiltrated subcutaneously in inverted L block. Sedation was not used.

Ljubljana method - percutaneous omentopexy using Bühner tape (silk ribbon)

Standing laparotomy was performed in right paralumbar fossa. Vertical or slightly oblique cranio-caudal surgical incision started little less than a hand-width beneath transverse processes of lumbar vertebrae and continued until the most caudal part of the costal arch where it ended about 4 to $5 \mathrm{~cm}$ caudal from it. M. obliqus abdominis externus and internus were incised, although internus could be also separated bluntly in the direction of muscle fibres. For entering the abdomen, rat-tooth tissue forceps were used to tent fascia and peritoneum, and blunt Mayo scissors for cutting a hole big enough for passing a finger that helped as a guide for extending the incision with scissors dorsally and ventrally. When the abdomen was entered, the suction of air into abdominal cavity could be heard. For haemostasis Pean haemostats and $4 \times 4$ s were used. The surgical incision should be long enough to permit passing a hand up to a shoulder. During the abdomen exploration, the quantity and quality of abdominal fluid was evaluated followed by examination of the kidneys, caecum, uterus, small and large intestines, rumen, and displaced abomasum. Then the abomasum was deflated at its most dorsal part at an acute angle with a $2 \mathrm{~mm}$ diameter needle attached to sterile rubber tubing until there was no palpable gas left in it. Palpation of the cranial part of the abdomen was followed by evaluation of liver fattiness, gall bladder, omasum and reticulum. Thick omentum close to pylorus was then grabbed and the abomasum pulled to the right side in case of left DA. In right DA or abomasal volvulus, the abomasum was deflated after excluding the possibility of volvulus, perforating ulcer or establishing the direction of volvulus followed by retorsion and before any exploration of the abdomen. Identification of pylorus was the next step. The landmark was thick omentum, which was followed cranio-ventrally until pylorus was reached. Pylorus (Plate II, Fig. 1) could be identified due to its unique characteristics: pale bluish pink colour, distinctive difference from omentum majus and its meaty structure on palpation. For omentopexy silk ribbon $(1 \mathrm{~cm}$ wide and $50 \mathrm{~cm}$ long), originally used for Bühner closure of vulva in cows, was used and placed with Gerlach needle (Plate II, Fig. 2). Omentopexy suture was positioned about 5 
to $10 \mathrm{~cm}$ caudal from pylorus through the thick omentum with Gerlach needle. Than each loose end of a suture was passed through the abdominal wall using Gerlach needle about $5 \mathrm{~cm}$ beneath the ventral end of the surgical incision (Plate III, Fig. 3). To enable penetration of the needle through the skin, small stab incision was made over the protruding Gerlach needle. The same was repeated with the other loose end of the omentopexy suture. Position of the abomasum was rechecked and both ends of the suture tightened (not too tight in order to prevent ischemia of the omentum). The suture was supported with rolled sterile $4 \times 4$, which was put under the knot. Surgical incision was closed with three layers. Peritoneum, fascia and transversus muscle were sutured together with simple continuous pattern with chromic catgut. M. obliqus internus if bluntly separated was apositioned with about 3 simple interrupted sutures and $\mathrm{m}$. obliqus externus was sutured with simple continuous pattern, both with chromic catgut. Skin was closed with supramid (Ford interlocking suture and most ventral 2 sutures were simple interrupted). Skin sutures and omentopexy sutures were removed about 10 days after the surgery.

\section{Results}

Reduced appetite or off feed, reduced milk production, not chewing cud and reduced faecal output of more dry or pasty faeces were recorded in all cows. Other predisposing factors for abomasum displacement like obesity, milk fever, retained placenta etc. were found. Concurrent diseases were often present (Table 1). Ketosis that did not respond favourably to treatment was very common.

Table 1. Information on concurrent diseases in treated cows using Ljubljana method at the hospital and their follow up status after 1 month

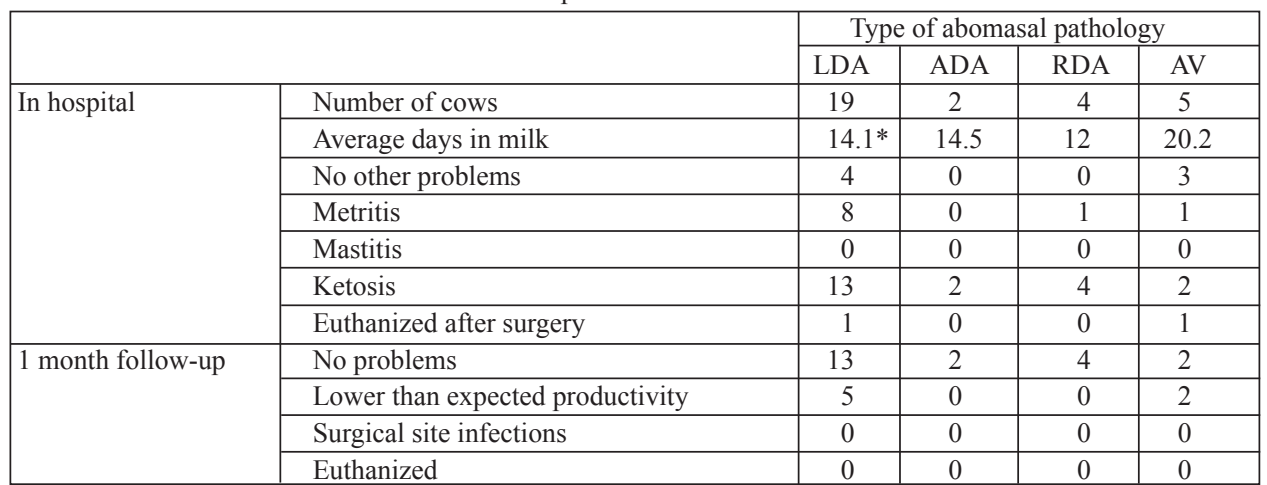

*One cow had LDA 140 days in milk and was not included in this calculation

LDA - Left displacement of abomasum, RDA - Right displacement of abomasum, ADA - Anterior displacement of abomasum, AV - Abomasal volvulus

Clinical examination usually revealed typical ping effect on simultaneous percussion and auscultation positioned either in the right or left costal region of abdomen that could extend to paralumbar fossa, depressed ruminal motor activity, often mild jaundice, deep paralumbar fossa, positive succussion test over sight of abomasal displacement (splashing sounds), sound of gas bubbles emerging through accumulated fluid in the abomasum on auscultation, high or slightly elevated arterial pulse and often enophtalmus (especially if diagnosed late in the course of the disease). Clinicopathological findings indicative of abomasal displacement included hypokalaemia, hypochloridaemia, hyperbilirubinaemia, mild hypocalcaemia, elevated beta-hydroxy butyrate. Higher activities of aspartame amino transferase, lactate dehydrogenase, gamma glutamyl transferase and glutamate dehydrogenase were frequently observed. Stress leucogram and hyperglycaemia were also present in many cases. Especially in long-lasting and AV cases, haemoconcentration was often observed. Clinical signs were usually more pronounced in abomasal volvulus (AV) cases, where also signs of dehydration were usually more evident. 
The surgical correction was successful in 28 cows, 2 had to be euthanized, one due to bad clinical status after abomasum displacement and perforating ulcer correction and the other due to $\mathrm{n}$. vagus indigestion following AV correction. No relapses occurred within a month after the surgery. All 28 cows were productive one month after the surgery.

\section{Discussion}

Since the first cow was treated for DA at the Clinic for Ruminants, Veterinary Faculty of Ljubljana in 1969 (Skušek et al. 1970), the frequency of the disease has increased rapidly (Zadnik et al. 2001). Based on recent data collected by Veterinary Administration of the Republic of Slovenia, current incidence rate of DA is about $2 \%$ of dairy cows in lactation. Displaced abomasum is also the most frequent diagnosis in dairy cows admitted at the Clinic for Ruminants.

Clinical and clinic-pathological findings in studied cows with abomasal displacement were in accordance with findings of other studies (Zadnik et al. 2001; Zadnik 2003b).

Experience with DA and AV shows that the clinical treatment using Ljubljana method is successful. Based on our experience, percutaneous omentopexy using Ljubljana method does not produce permanent adhesion of the omentum to the abdominal wall. When omentopexy suture is removed about 7-14 days after the surgery, abomasum can be free again and can move in the abdominal cavity. We assume that in the period of up to 14 days after the surgery, the rumen improves to disable abomasal movement. Affected cows also stabilize metabolically which is necessary for the healthy tonus and motorical function of the abomasum. Even though abomasum may be free when the omentopexy suture is released, re-displacements are very rare, and none was observed in cows included in this study. We did not perform follow-up ultrasound or laparoscopic examination to exclude formation of permanent adhesions after Ljubljana method surgical correction of DA. Our assumption is based on few documented cases of cows $(n=15)$ which had DA again in consecutive lactations. A small round adhesion of about $0.5 \mathrm{~cm}$ diameter and a few $\mathrm{cm}$ length of the omentum to the abdominal wall was observed only once in these cows. Even in a cow that was surgically treated for LDA in the second, third and fourth lactation, there were no adhesions found at the surgery.

The possibility of re-displacement is a disadvantage of this method compared to methods that produce a permanent adhesion. This is the reason why some owners of cows with DA prefer permanent fixation. However, we are of the opinion that enabling normal physiological movement of the abomasum during pregnancy is beneficial for cows. Permanent omentopexy can cause stretching of the omentum in late pregnancy when the abomasum moves physiologically more cranial and transverse than in non-pregnant cows (Wittek et al. 2005). The situation can be more aggravated in cows with abomasopexy. This can potentially cause discomfort and complications when these cows are in late pregnancy. Another potential disadvantage of surgical correction of DA using Ljubljana method compared to other surgical methods that do not produce percutaneous pexy is the possibility of infection through percutaneous omentopexy suture. Omentopexy suture presents a potential route of entry for bacteria into the abdominal cavity. This could be a problem especially if cows are kept in unsanitary conditions after surgery. At the Clinic for Ruminants in Ljubljana, cows are hospitalized for about 3 to 7 days after surgery and receive antibiotic treatment. If omentopexy suture is soiled it needs to be cleaned and disinfected (we usually use povidon iodide or oxytetracycline spray). Occasionally some pus comes out of the suture site after the suture is removed, but infection usually resolves spontaneously without complication. To reduce the possibility of abdominal contamination and infection, the suture should be disinfected with povidon iodide and then pulled lightly before cutting one side of it and pulling it out. In case of fluid accumulation in the abdominal cavity, omentopexy suture draining peritoneal fluid was observed. 
Surgical treatment of DA using Ljubljana method is financially acceptable and useful for all types of DA and AV. Bühner tape is not expensive, and except the Gerlach needle there are no other special surgical instruments needed. Because only one percutaneous omentopexy suture is placed, the surgery can be completed faster than in laparotomy techniques where pexy is carried out by suturing. The surgery can be performed also in the field. Many ambulatory cattle practitioners in Slovenia successfully perform it on farms.

In conclusion, we recommend omentopexy using Ljubljana method as an alternative to laparoscopy-guided surgery and other laparotomy techniques, as a clinical procedure as well as procedure performed in ambulatory practice. The surgery can be used in all types of DA and AV; it is rapidly performed in standing animal; it does not need expensive equipment; it can be performed with minimal personnel; it has low recurrence rate, and is not expensive. It can also be used in cows in late pregnancy.

\section{Acknowledgment}

The authors thank Jožica Ježek PhD, DVM and Čedo Bursač, veterinary technician, for their help in cow treatment and Marija Nemec MSc, DVM for performing haematology and biochemistry analyses.

\section{References}

Constable PD, Miller GY, Hoffsis GF, Hull BL, Rings DM 1992: Risk factors for abomasal volvulus and left abomasal displacement in cattle. Am J Vet Res 53: 1184-1191

Rohn M, Tenhagen B-A, Hofmann W 2004: Survival of dairy cows after surgery to correct abomasal displacement: 2. Association of clinical and laboratory parameters with survival in cows with left abomasal displacement. $\mathrm{J}$ Vet Med Series A 51: 300-305

Roy JP, Harvey D, Bélanger AM, Buczinski S 2008: Comparison of 2-step laparoscopy-guided abomasopexy versus omentopexy via right flank laparotomy for the treatment of dairy cows with left displacement of the abomasum in on-farm settings. J Am Vet Med Assoc 232: 1700-1706

Seeger T, Kümper H, Failing K, Doll K 2006: Comparison of laparoscopic-guided abomasopexy versus omentopexy via right flank laparotomy for the treatment of left abomasal displacement in dairy cows. Am J Vet Res 67: 472-478

Skušek F, Gregorović V, Jazbec I 1970: Right and then left displacement of the abomasum in a cow. Vet Glasn 24: 285

Steiner A 2006: Surgical treatment of the left displacement of the abomasum an update. In: XXIV. World Buiatrics Congress, Nice, France, pp. 165-169

Wittek T, Constable PD, Morin DE 2005: Ultrasonographic assessment of change in abomasal position during the last three months of gestation first three months of lactation in Holstein-Friesian cows. J Am Vet Med Assoc 227: $1469-1475$

Wittek T, Sen I, Constable PD 2007: Changes in abdominal dimensions during late gestation and early lactation in Holstein-Friesian heifers and cows and their relationship to left displaced abomasum. Vet Rec 161: 155-161

Zadnik T, Mesarič M, Reichel P 2001: A review of abomasal displacement - clinical and laboratory experiences at the Clinic for ruminants in Ljubljana. Slov Vet Res 38: 201-216

Zadnik T 2003a: Review of anterior displacement of the abomasum in cattle in Slovenia. Vet Rec 153: 24-25

Zadnik T 2003b: A comparative study of the hemato-biochemical parameters between clinically healthy cows and cows with displacement of the abomasum. Acta Vet Beograd 53: 297-309 

Plate II

Starič J. et al.: Surgical ... pp. 469-473

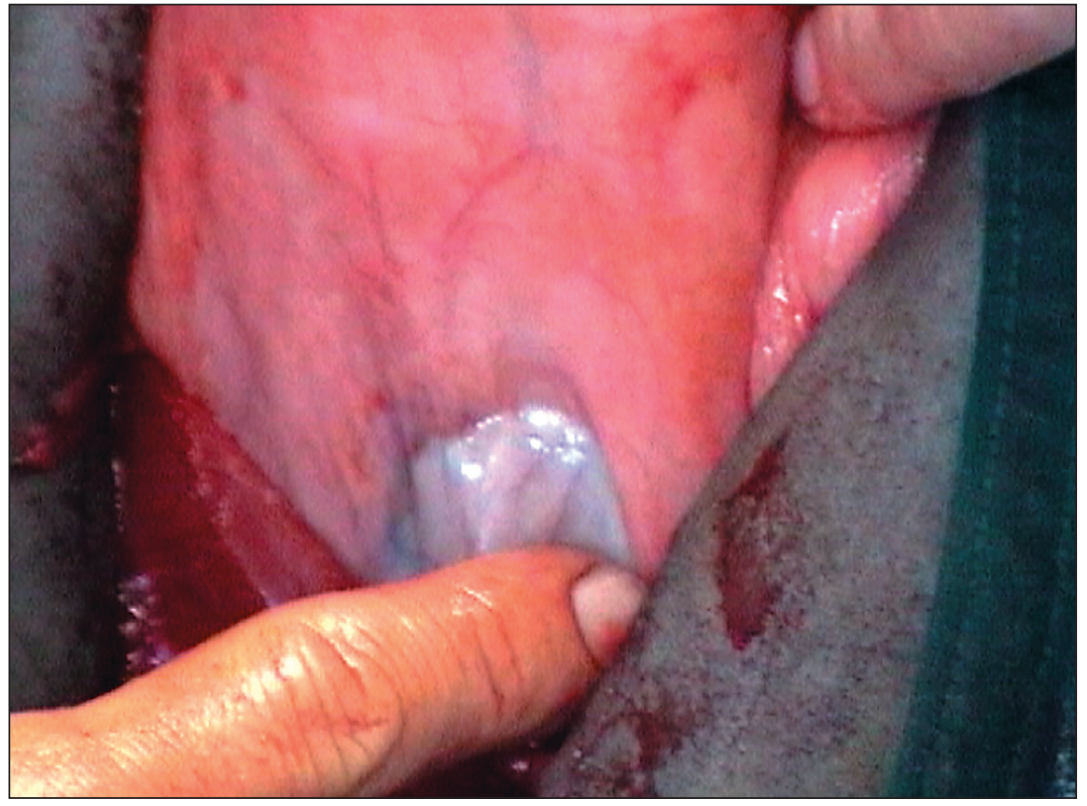

Fig. 1. Appearence of pylorus during operation

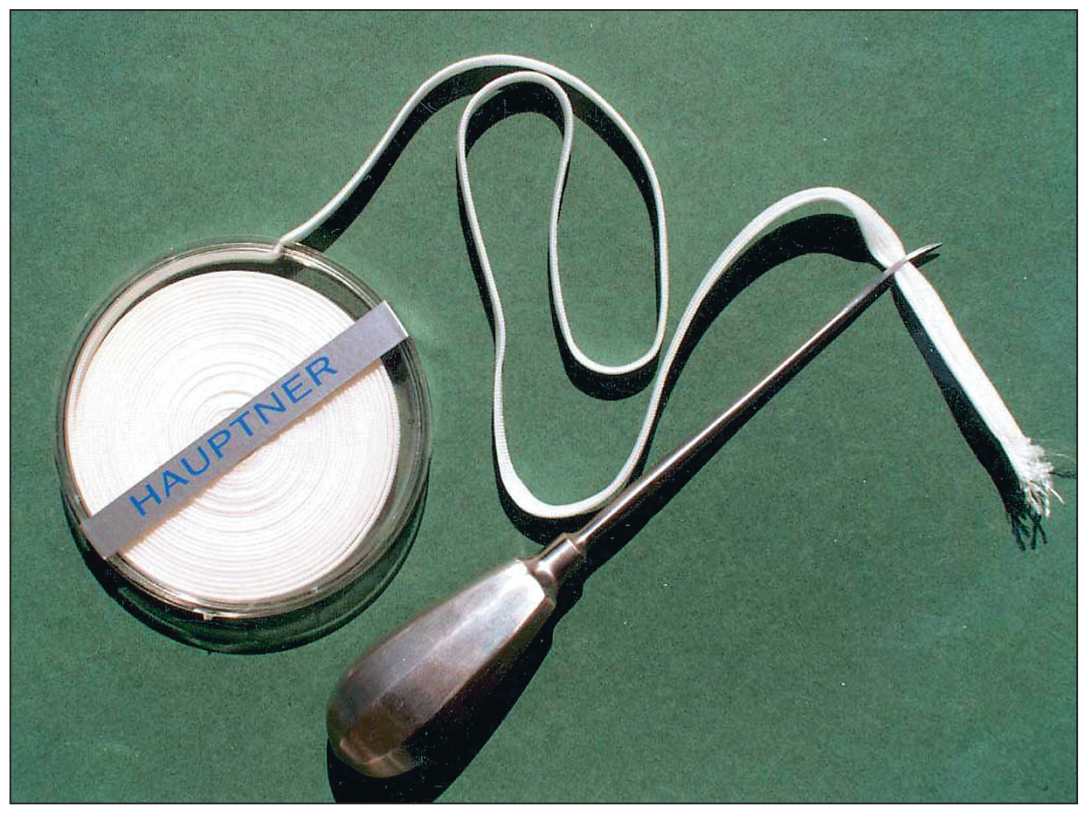

Fig. 2. Bühner tape and Gerlach needle 
Plate III

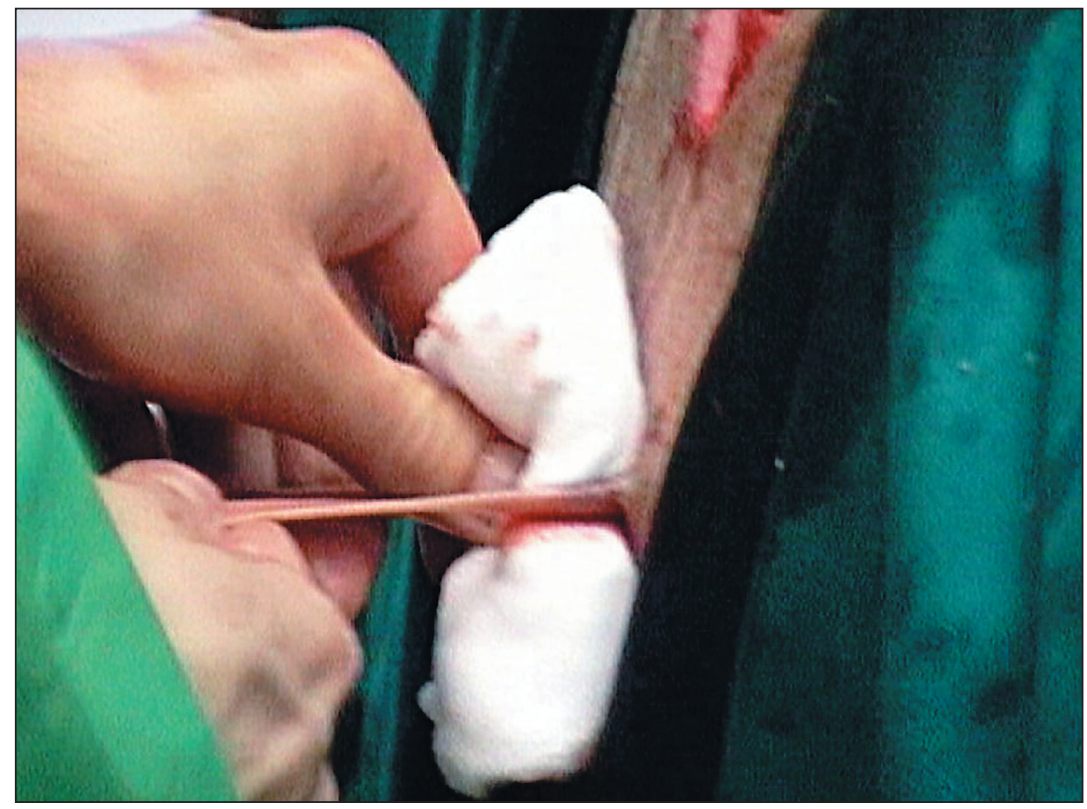

Fig. 3. End of suture is passed through the abdominal wall using Gerlach needle about $5 \mathrm{~cm}$ beneath the ventral end of the surgical incision 